\title{
What Makes the Arc-Preserving Subsequence Problem Hard?^
}

\author{
Guillaume Blin ${ }^{1}$, Guillaume Fertin ${ }^{1}$, Romeo Rizzi ${ }^{2}$, and Stéphane Vialette ${ }^{3}$ \\ 1 LINA - FRE CNRS 2729 Université de Nantes, \\ 2 rue de la Houssinière BP 9220844322 Nantes Cedex 3 - France \\ \{blin, fertin\}@univ-nantes.fr \\ 2 Università degli Studi di Trento Facoltà di Scienze - Dipartimento di Informatica e \\ Telecomunicazioni \\ Via Sommarive, 14 - I38050 Povo - Trento (TN) - Italy \\ Romeo.Rizzi@unitn.it \\ 3 LRI - UMR CNRS 8623 Faculté des Sciences d'Orsay, Université Paris-Sud \\ Bât 490, 91405 Orsay Cedex - France \\ vialette@lri.fr
}

\begin{abstract}
Given two arc-annotated sequences $(S, P)$ and $(T, Q)$ representing RNA structures, the ArC-PRESERVing Subsequence (APS) problem asks whether $(T, Q)$ can be obtained from $(S, P)$ by deleting some of its bases (together with their incident arcs, if any). In previous studies [3, 6, this problem has been naturally divided into subproblems reflecting intrinsic complexity of arc structures. We show that APS(Crossing, Plain) is NP-complete, thereby answering an open problem [6]. Furthermore, to get more insight into where actual border of APS hardness is, we refine APS classical subproblems in much the same way as in 11 and give a complete categorization among various restrictions of APS problem complexity.
\end{abstract}

Keywords: RNA structures, Arc-Preserving Subsequence, Computational complexity.

\section{Introduction}

At a molecular state, the understanding of biological mechanisms is subordinated to RNA functions discovery and study. Indeed, it is established that the conformation of a single-stranded RNA molecule (a linear sequence composed of ribonucleotides $A, U, C$ and $G$, also called primary structure) partly determines the molecule function. This conformation results from the folding process due to local pairings between complementary bases $(A-U$ and $C-G)$. The RNA secondary structure is a collection of folding patterns that occur in it.

RNA secondary structure comparison is important in many contexts, such as (i) identification of highly conserved structures during evolution which suggest a significant common function for the studied RNA molecules [9], (ii) RNA

\footnotetext{
* This work was partially supported by the French-Italian PAI Galileo project number $08484 \mathrm{VH}$ and by the CNRS project ACI Masse de Données "NavGraphe".
} 
classification of various species (phylogeny) 2, (iii) RNA folding prediction by considering a set of already known secondary structures [13].

Structure comparison for RNA has thus become a central computational problem bearing many challenging computer science questions. At a theoretical level, RNA structure is often modelled as an arc-annotated sequence, that is a pair $(S, P)$ where $S$ is a sequence of ribonucleotides and $P$ represents hydrogen bonds between pairs of elements of $S$. Different pattern matching and motif search problems have been investigated in the context of arc-annotated sequences among which we can mention ArC-PrESERving SubSequence (APS) problem, Edit Distance problem, ArC-Substructure (AST) problem and Longest ArC-Preserving Subsequence (LAPCS) problem (see for instance 3, 8, 7, 6, 1). For other related studies concerning algorithmic aspects of (protein) structure comparison using contact maps, refer to [5, 10.

In this paper, we focus on APS problem: given two arc-annotated sequences $(S, P)$ and $(T, Q)$, this problem asks whether $(T, Q)$ can be exactly obtained from $(S, P)$ by deleting some of its bases together with their incident arcs, if any. This problem is commonly encountered when one is searching for a given RNA pattern in an RNA database 77. Moreover, from a theoretical point of view, APS problem can be seen as a restricted version of LAPCS problem, and hence has applications in structural comparison of RNA and protein sequences 3, 5, 12. APS problem has been extensively studied in the past few years 6, 7 3. Of course, different restrictions on arc-annotation alter APS computational complexity, and hence this problem has been naturally divided into subproblems reflecting the complexity of the arc structure of both $(S, P)$ and $(T, Q)$ : PLAIN, Chain, Nested, CRossing or Unlimited (see Section 2 for details). All of them but one have been classified as to whether they are polynomial time solvable or NP-complete. The problem of the existence of a polynomial time algorithm for APS(Crossing,Plain) problem was mentioned in [6] as the last open problem in the context of arc-preserving subsequences. Unfortunately, as we shall prove in Section 4 APS(Crossing,Plain) is NP-complete even for restricted special cases.

In analyzing the computational complexity of a problem, we are often trying to define a precise boundary between polynomial and NP-complete cases. Therefore, as another step towards establishing the precise complexity landscape of APS problem, we consider that it is of great interest to subdivide existing cases into more precise ones, that is to refine classical complexity levels of APS problem, for determining more precisely what makes the problem hard. For that purpose, we use the framework introduced by Vialette [11 in the context of 2intervals (a simple abstract structure for modelling RNA secondary structures). As a consequence, the number of complexity levels rises from 4 to 8 , and all the entries of this new complexity table need to be filled. Previous known results concerning APS problem, along with our NP-completeness proofs, allow us to fill all the entries of this new table, therefore determining what exactly makes the APS problem hard. 
The paper is organized as follows. Provided with notations and definitions (Section 2), in Section 3 we introduce and explain new refinements of the complexity levels we are going to study. In Section 4 , we show that $\operatorname{APS}(\{\sqsubset, \varnothing\}, \emptyset)$ is NP-complete thereby proving that classical APS(Crossing, Plain) is NPcomplete as well. As another refinement to that result, we prove that $\operatorname{APS}(\{<, \gamma$ \}$, \emptyset)$ is NP-complete. Finally, in Section 5, we give new polynomial time solvable algorithms for restricted instances of APS(Crossing, Plain).

\section{$2 \quad$ Preliminaries}

An RNA structure is commonly represented as an arc-annotated sequence $(S, P)$ where $S$ is a sequence of ribonucleotides (or bases) and $P$ is a set of arcs connecting pairs of bases in $S$. Let $(S, P)$ and $(T, Q)$ be two arc-annotated sequences such that $|S| \geq|T|$ (in the following, $n=|S|$ and $m=|T|$ ). APS problem asks whether $(T, Q)$ can be exactly obtained from $(S, P)$ by deleting some of its bases together with their incident arcs, if any.

Since the general problem is easily seen to be intractable [3], the arc structure must be restricted. Evans [3] proposed four possible restrictions on $P$ (resp. Q) which were largely reused in subsequent literature: (1) there is no base incident to more than one arc, (2) there are no arcs crossing, (3) there is no arc contained in another and (4) there is no arc.

These restrictions are used progressively and inclusively to produce five different levels of allowed arc structure: UNLIMITED - general problem with no restrictions, CRossing - restriction (1); NESTED - restrictions (1) and (2); CHAIN - restrictions (1), (2) and (3); PLAIN - restriction (4).

Guo proved in [7] that APS(Crossing, Chain) is NP-complete. Guo et al. observed in [6] that NP-completeness of APS(Crossing, Crossing) and APS(Unlimited, Plain) easily follows from results of Evans [3] concerning LAPCS problem. Furthermore, they gave a $O(\mathrm{~nm})$ time algorithm for APS (Nested, Nested). This algorithm can be applied to easier problems such as APS(Nested, $\alpha)$ and $\operatorname{APS}(\mathrm{Chain}, \alpha)$ with $\alpha \in\{$ Chain,Plain $\}$. Finally, Guo et al. mentioned in [6] that APS(ChAIN, PlaIN) can be solved in $O(n+m)$ time. Observe that UNLIMITED level has no restrictions, and hence is of limited interest in our study. Consequently, from now on we will not be concerned anymore with that level. Until now, the question of the existence of an exact polynomial algorithm for APS(Crossing, Plain) remained open. We will show in the present paper that APS(Crossing,Plain) is NP-complete.

\section{Refinement of APS Problem}

In this section, we propose a refinement of APS problem. We first state formally our approach and explain why such a refinement is relevant for both theoretical and experimental studies. 


\subsection{Splitting the Levels.}

As we will show soon, APS(Crossing, Plain) is NP-complete. That result answers the last open problem concerning APS computational complexity with respect to classical complexity levels. However, we are mainly interested in the elaboration of a precise border between NP-complete and polynomially solvable cases. Indeed, both theorists and practitioners might naturally ask for more information concerning APS hard cases in order to get valuable insight into what makes the problem difficult. As a next step towards better understanding what makes APS problem hard, we propose to refine classically models used for classifying arc-annotated sequences. Our refinement consists in splitting those models of arc-annotated sequences into more precise relations between arcs. For example, such a refinement provides a general framework for investigating polynomial time solvable and hard restricted instances of APS(Crossing, Plain), thereby refining in many ways Theorem 1 (see Section 5 ).

We use three relations first introduced by Vialette 11] in the context of 2intervals. Actually, his definition of 2-intervals could almost apply in this paper (the main difference lies in the fact that 2-intervals are used for representing sets of contiguous arcs). Vialette defined three possible relations between 2-intervals that can be used for arc-annotated sequences as-well. They are the following: for any two $\operatorname{arcs} A_{1}=(i, j)$ and $A_{2}=(k, l)$ in $P$, we will write $A_{1}<A_{2}$ if $i<j<k<l$ (precedence relation), $A_{1} \sqsubset A_{2}$ if $k<i<j<l$ (nested relation) and $A_{1} \gamma A_{2}$ if $i<k<j<l$ (crossing relation). Two arcs $A_{1}$ and $A_{2}$ are $\tau$-comparable for some $\tau \in\{<, \sqsubset, \varnothing\}$ if $A_{1} \tau A_{2}$ or $A_{2} \tau A_{1}$. Let $\mathcal{P}$ be a set of arcs and $R$ be a non-empty subset of $\{<, \sqsubset, \chi\}$. The set $\mathcal{P}$ is said to be $R$-comparable if any two distinct arcs of $\mathcal{P}$ are $\tau$-comparable for some $\tau \in R$. An arc-annotated sequence $(P, A)$ is said to be an $R$-arc-annotated sequence for some non-empty subset $R$ of $\{<, \sqsubset, \varnothing\}$ if $A$ is $R$-comparable. By abuse of notation, we write $R=\emptyset$ in case $A=\emptyset$. Observe that our model cannot deal with arc-annotated sequences which contain only one arc. However, having only one arc or none can not really affect the problem computational complexity. Just one guess reduces from one case to the other.

As a straightforward illustration of above definitions, classical complexity levels for APS problem can be expressed in terms of combinations of our new relations: Plain is fully described by $R=\emptyset$, Chain by $R=\{<\}$, Nested by $R=\{<, \sqsubset\}$ and Crossing by $R=\{<, \sqsubset, \chi\}$. The key point is to observe that our refinement allows us to consider new structures for arc-annotated sequences, namely $R \in\{\{\sqsubset\},\{\chi\},\{<, \chi\},\{\sqsubset, \chi\}\}$, which could not be considered using classical complexity levels. Although other refinements may be possible (in particular well-suited for parameterized complexity analysis), we do believe that such an approach allows a more precise analysis of APS complexity. Of course one might object that some of these subdivisions are unlikely to appear in RNA secondary structures. However, it is of great interest to answer, at least partly, the following question: Where is the precise boundary between polynomial and NP-complete cases? Indeed, such a question is relevant for both theoretical and experimental studies. 


\subsection{Immediate Results}

First, observe that we only have to consider cases of $\operatorname{APS}\left(R_{1}, R_{2}\right)$ where $R_{1}$ and $R_{2}$ are compatible, i.e. $R_{2} \subseteq R_{1}$. Indeed, if this is not the case, we can immediately answer negatively since there exists two arcs in $T$ which satisfy a relation in $R_{2}$ which is not in $R_{1}$, and hence $T$ simply cannot be obtained from $S$ by deleting bases of $S$. Those useless cases are simply denoted by hatched areas in Table 1.

Some known results allow us to fill many entries of the new complexity table derived from our refinement. The remainder of this subsection is devoted to detailing these first easy statements. We begin with an easy observation concerning complexity propagation properties of APS problem.

Observation 1 Let $R_{1}, R_{2}, R_{1}^{\prime}$ and $R_{2}^{\prime}$ be four subsets of $\{<, \sqsubset, \gamma\}$ such that $R_{2}^{\prime} \subseteq R_{2} \subseteq R_{1}$ and $R_{2}^{\prime} \subseteq R_{1}^{\prime} \subseteq R_{1}$. If $\operatorname{APS}\left(R_{1}^{\prime}, R_{2}^{\prime}\right)$ is NP-complete (resp. $\operatorname{APS}\left(R_{1}, R_{2}\right)$ is polynomial time solvable) then so is $\operatorname{APS}\left(R_{1}, R_{2}\right)$ (resp. $\left.\operatorname{APS}\left(R_{1}^{\prime}, R_{2}^{\prime}\right)\right)$.

On the positive side, Guo et al. have shown that APS(NESTED, NESTED) is solvable in $O(\mathrm{~nm})$ time [6]. Another way of stating this is to say that $\operatorname{APS}(\{<$ $, \sqsubset\},\{<, \sqsubset\})$ is solvable in $O(m n)$ time. That result together with Observation 1 may be summarized by saying that $\operatorname{APS}\left(R_{1}, R_{2}\right)$ for any compatible $R_{1}$ and $R_{2}$ such that $\gamma \notin R_{1}$ and $\gamma \notin R_{2}$ is polynomial time solvable.

Conversely, Evans has proved that APS(Crossing, Crossing) is NP-complete [3. A simple reading shows that her proof is concerned with $\{<, \sqsubset, \varnothing\}$-arcannotated sequences, and hence she actually proved that $\operatorname{APS}(\{<, \sqsubset, \gamma\},\{<, \sqsubset$ $,\{\})$ is NP-complete. Similarly, in proving that APS(Crossing, Chain) is NPcomplete [7, Guo actually proved that $\operatorname{APS}(\{<, \sqsubset, \gamma\},\{<\})$ is NP-complete. Note that according to Observation 1, this latter result implies that $\operatorname{APS}(\{<$ $, \sqsubset, \gamma\},\{<, \sqsubset\})$ and $\operatorname{APS}(\{<, \sqsubset, \gamma\},\{<, \varnothing\})$ are $\mathbf{N P}$-complete. Table 1 surveys known and new results for various types of our refined APS problem. Observe that this paper answers all questions concerning APS problem with respect to both classical and new complexity levels.

Table 1. Complexity results after complexity levels refinement. ////: useless cases. $\star$ : results from this paper.

\begin{tabular}{|c|c|c|c|c|c|c|c|}
\hline \multicolumn{8}{|c|}{ APS } \\
\hline$R_{1} \backslash R_{2}$ & \begin{tabular}{|l|l|}
$\{<, \sqsubset, \varnothing\}$ & $\{\sqsubset, \varnothing\}$ \\
\end{tabular} & $\{<, \varnothing\}$ & $\{\chi\}$ & $\{<, \sqsubset\}$ & $\{\sqsubset\}$ & $\{<\}$ & $\emptyset$ \\
\hline$\{<, \sqsubset, \varnothing\}$ & $|\mathbf{N P}-\mathrm{C} 3| \mid \mathbf{N P}-\mathrm{C} \star$ & $\mid$ NP-C 7 & NP-C * & \begin{tabular}{ll|} 
NP-C & 7 \\
\end{tabular} & NP-C $\star$ & \begin{tabular}{ll|} 
NP-C 7 & \\
\end{tabular} & NP-C $\star$ \\
\hline$\{\sqsubset, \chi\}$ & NP-C $\star$ & $1 / / /$ & NP-C $\star$ & $/ / / /$ & NP-C $\star$ & $1 / / /$ & NP-C $\star$ \\
\hline$\{<, \chi\}$ & & NP-C $\star$ & $\mathrm{NP}-\mathrm{C} \star$ & $/ / / /$ & $1 / / /$ & $\mathrm{NP}-\mathrm{C} \star$ & NP-C $\star$ \\
\hline$\{\chi\}$ & & & $O\left(n m^{2}\right) \star$ & $1 / / / 1$ & $1 / / / 1$ & $/ / / /$ & $O\left(n m^{2}\right) \star$ \\
\hline$\{<, \sqsubset\}$ & & & & $O(n m) 6$ & O(nm) 6 & $O(n m) 6$ & $O(n m) 6$ \\
\hline$\{\sqsubset\}$ & & & & & $O(n m) \quad 6$ & $1 / / /$ & $O(\mathrm{~nm}) 6$ \\
\hline$\{<\}$ & & & & & & $O(n m) \quad 6$ & $O(n+m) \quad 6$ \\
\hline $\bar{\emptyset}$ & & & & & & & $\overline{O(n+m) \underline{6}}$ \\
\hline
\end{tabular}




\section{$4 \quad$ Hardness Results}

We show in this section that $\operatorname{APS}(\{\sqsubset, \varnothing\}, \emptyset)$ and $\operatorname{APS}(\{<, \varnothing\}, \emptyset)$ are NP-complete thereby proving that APS(Crossing, Plain) is NP-complete. That result answers an open problem posed by Gramm, Guo and Niedermeier in [6] which is the last open problem concerning the APS computational complexity with respect to classical complexity levels, i.e., Plain, Chain, Nested and Crossing.

Theorem 1. $\operatorname{APS}(\{\sqsubset, \varnothing\}, \emptyset)$ is NP-complete.

We prove Theorem 1 by giving a polynomial time reduction from the well known NP-complete 3-SAT problem [4. Due to space considerations, the entire proof is deferred to the full version of the paper.

It follows immediately from Theorem 1 that $\operatorname{APS}(\{<, \sqsubset, \gamma\}, \emptyset)$, and hence APS(Crossing, Plain), are NP-complete. One might naturally ask for more information concerning hard cases of APS problem in order to get valuable insight into what makes the problem difficult. Another refinement of the hardness of APS(Crossing,Plain) is given by the following theorem.

Theorem 2. $\operatorname{APS}(\{<, \varnothing\}, \emptyset)$ is $\mathbf{N P}$-complete.

The proof is also by reduction from 3-SAT. Due to space considerations, the rather technical proof is also deferred to the full version of the paper.

\section{Two Polynomial Time Solvable APS Problems}

We prove in this section that $\operatorname{APS}(\{\gamma\}, \emptyset)$ and $\operatorname{APS}(\{\gamma\},\{\gamma\})$ are polynomial time solvable. In other words, relation $\chi$ alone does not imply NP-completeness. We need the following notations. Sequences are the concatenation of zero or more elements from an alphabet. We use the period "." as the concatenation operator, but frequently the two operands are simply put side by side. Let $T=$ $T[1] T[2] \ldots T[m]$ be a sequence of length $m$. For all $1 \leq i \leq j \leq m$, we write $T[i: j]$ to denote $T[i] T[i+1] \ldots T[j]$. The reverse of $T$ is the sequence $T^{R}=$ $T[m] \ldots T[2] T[1]$. A factorization of $T$ is any decomposition $T=x_{1} x_{2} \ldots x_{q}$ where $x_{1}, x_{2}, \ldots x_{q}$ are (possibly empty) sequences. Let $(T, A)$ be a $\{\gamma\}$-arcannotated sequence and $(i, j) \in A, i<j$, be an arc. We call $T[i]$ a forward base and $T[j]$ a backward base. We will denote by $L_{T}$ the position of the last forward base in $(T, A)$ and by $\mathrm{FB}_{T}$ the position of the first backward base in $(T, A)$, i.e., $\mathrm{LF}_{T}=\max \{i:(i, j) \in A\}$ and $\mathrm{FB}_{T}=\min \{j:(i, j) \in A\}$. By convention, we let $\mathrm{LF}_{T}=0$ and $\mathrm{FB}_{T}=|T|+1$ if $A=\emptyset$. Observe that $\mathrm{LF}_{T}<\mathrm{FB}_{T}$. We begin by proving a factorization result on $\{\gamma\}$-arc-annotated sequences.

Lemma 1. Let $S$ and $T$ be two $\{\gamma\}$-arc-annotated sequences of length $n$ and $m$, respectively. If $T$ occurs as an arc preserving subsequence in $S$, then there exists a factorization (possibly trivial) $T\left[\mathrm{LF}_{T}+1: \mathrm{FB}_{T}-1\right]=x y$ such that $T[1$ : $\left.\mathrm{LF}_{T}\right] \cdot x \cdot\left(y \cdot T\left[\mathrm{FB}_{T}: m\right]\right)^{R}$ occurs as an arc preserving subsequence in $S[1$ : $\left.\mathrm{FB}_{S}-1\right] \cdot S\left[\mathrm{FB}_{S}: n\right]^{R}$. 
Proof. Suppose that $T$ occurs as an arc preserving subsequence in $S$. Since both $S$ and $T$ are $\{\gamma\}$-arc-annotated sequences, then there exist two factorizations $S\left[1: \mathrm{LF}_{S}\right]=u w$ and $S\left[\mathrm{FB}_{S}: n\right]=z v$ such that: (i) $T\left[1: \mathrm{LF}_{T}\right]$ occurs in $u$, (ii) $T\left[\mathrm{LF}_{T}+1: \mathrm{FB}_{T}-1\right]$ occurs in $w \cdot S\left[\mathrm{LF}_{S}+1: \mathrm{FB}_{S}-1\right] \cdot z$ and (iii) $T\left[\mathrm{FB}_{T}\right.$ : $m]$ occurs in $v$. Then it follows that there exists a factorization $T\left[\operatorname{LF}_{T}+1\right.$ : $\left.\mathrm{FB}_{T}-1\right]=x y$ such that $x$ occurs in $w \cdot S\left[\mathrm{LF}_{S}+1: \mathrm{FB}_{S}-1\right]$ and $y$ occurs in $z$, and hence $T^{\prime}=T\left[1: \mathrm{LF}_{T}\right] \cdot x \cdot\left(y \cdot T\left[\mathrm{FB}_{T}: m\right]\right)^{R}$ occurs as an arc preserving subsequence in $S^{\prime}=S\left[1: \mathrm{FB}_{S}-1\right] \cdot S\left[\mathrm{FB}_{S}: n\right]^{R}$

Theorem 3. APS $(\{\chi\},\{\gamma\})$ is solvable in $O\left(n m^{2}\right)$ time.

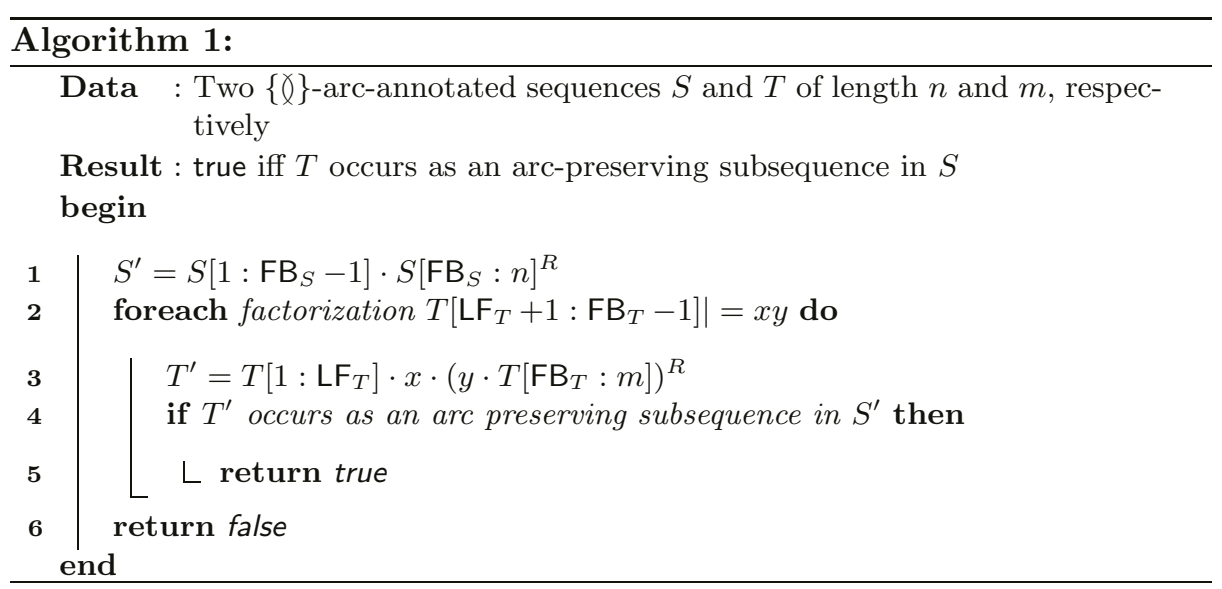

Proof. Consider Algorithm 1. Correctness of the algorithm follows from Lemma 1. What is left is to prove the time complexity. Clearly, $S^{\prime}=S\left[1: \mathrm{FB}_{S}-1\right] \cdot S\left[\mathrm{FB}_{S}\right.$ : $n]^{R}$ is a $\{\sqsubset\}$-arc-annotated sequence. The key point is to note that, for any factorization $T\left[\mathrm{LF}_{T}+1: \mathrm{FB}_{T}-1\right] \mid=x y$, the obtained $T^{\prime}=T\left[1: \mathrm{LF}_{T}\right] \cdot x \cdot\left(y \cdot T\left[\mathrm{FB}_{T}\right.\right.$ : $m])^{R}$ is a $\{\sqsubset\}$-arc-annotated sequence as-well. Now let $k$ be the number of arcs in $T$. So there are at most $m-2 k$ iterations to go before eventually returning false. According to the above, Line 4 constitutes an instance of $\operatorname{APS}(\{\sqsubset\},\{\sqsubset\})$. But $\operatorname{APS}(\{\sqsubset\},\{\sqsubset\})$ is a special case of $\operatorname{APS}(\{<, \sqsubset\},\{<, \sqsubset\})$, and hence is solvable in $O(n m)$ time [6]. Then it follows that the algorithm as a whole runs in $O(n m(m-2 k))=O\left(n m^{2}\right)$ time.

Clearly, the proof of Theorem 3 relies on an efficient algorithm for solving $\operatorname{APS}(\{\sqsubset\},\{\sqsubset\})$ : the better the complexity for $\operatorname{APS}(\{\sqsubset\},\{\sqsubset\})$, the better the complexity for $\operatorname{APS}(\{\chi\},\{\gamma\})$. We have used only the fact that $\operatorname{APS}(\{\sqsubset\},\{\sqsubset\})$ is a special case of $\operatorname{APS}(\{<, \sqsubset\},\{<, \sqsubset\})$. It remains open, however, wether a better complexity can be achieved for $\operatorname{APS}(\{\sqsubset\},\{\sqsubset\})$. Theorem 3 , combined with Observation 1, carries out easily to restricted versions.

Corollary 1. APS $(\{\chi\}, \emptyset)$ is solvable in $O\left(\mathrm{~nm}^{2}\right)$ time. 


\section{Conclusion}

In this paper, we investigated the APS problem time complexity and gave a precise characterization of what makes the APS problem hard. We proved that APS(Crossing,Plain) is NP-complete thereby answering an open problem posed in [6]. Note that this result answers the last open problem concerning APS computational complexity with respect to classical complexity levels, i.e., PLAIN, Chain, Nested and Crossing. Also, we refined the four above mentioned levels for exploring the border between polynomial time solvable and NP-complete problems. We proved that both $\operatorname{APS}(\{\sqsubset, \varnothing\}, \emptyset)$ and $\operatorname{APS}(\{<, \varnothing\}, \emptyset)$ are NPcomplete and gave positive results by showing that $\operatorname{APS}(\{\chi\}, \emptyset)$ and $\operatorname{APS}(\{\chi$ \}$,\{\chi\})$ are polynomial time solvable. Hence, the refinement we suggest shows that APS problem becomes hard when one considers sequences containing $\{\varnothing, \alpha\}$ comparable arcs with $\alpha \neq \emptyset$. Therefore, crossing arcs alone do not imply APS hardness. It is of course a challenging problem to further explore the complexity of the APS problem, and especially the parameterized views, by considering additional parameters such as the cutwidth or the depth of the arc structures.

\section{References}

1. J. Alber, J. Gramm, J. Guo, and R. Niedermeier. Computing the similarity of two sequences with nested arc annotations. Theoretical Computer Science, 312(23):337-358, 2004.

2. G. Caetano-Anollés. Tracing the evolution of RNA structure in ribosomes. Nucl. Acids. Res., 30:2575-2587, 2002.

3. P. Evans. Algorithms and Complexity for Annotated Sequence Analysis. PhD thesis, U. Victoria, 1999.

4. M. Garey and D. Johnson. Computers and Intractability: A Guide to the Theory of NP-Completeness. W. H. Freeman and Company, 1979.

5. D. Goldman, S. Istrail, and C.H. Papadimitriou. Algorithmic aspects of protein structure similarity. In Proc. of the 40th Symposium of Foundations of Computer Science (FOCS99), pages 512-522, 1999.

6. J. Gramm, J. Guo, and R. Niedermeier. Pattern matching for arc-annotated sequences. In Proc. of the 22nd Conference on Foundations of Software Technology and Theoretical Computer Science (FSTTCS02), volume 2556 of LNCS, pages 182193, 2002.

7. J. Guo. Exact algorithms for the longest common subsequence problem for arcannotated sequences. Master's Thesis, Universitat Tubingen, Fed. Rep. of Germany, 2002.

8. T. Jiang, G.-H. Lin, B. Ma, and K. Zhang. The longest common subsequence problem for arc-annotated sequences. In Proc. 11th Symposium on Combinatorial Pattern Matching (CPM00), volume 1848 of LNCS, pages 154-165. Springer-Verlag, 2000.

9. V. Juan, C. Crain, and S. Wilson. Evidence for evolutionarily conserved secondary structure in the H19 tumor suppressor RNA. Nucl. Acids. Res., 28:1221-1227, 2000. 
10. G. Lancia, R. Carr, B. Walenz, and S. Istrail. 101 optimal PDB structure alignments: a branch-and-cut algorithm for the maximum contact map overlap problem. In Proceedings of the 5th ACM International Conference on Computational Molecular Biology (RECOMB01), pages 193-202, 2001.

11. S. Vialette. On the computational complexity of 2-interval pattern matching. Theoretical Computer Science, 312(2-3):223-249, 2004.

12. K. Zhang, L. Wang, and B. Ma. Computing the similarity between RNA structures. In Proc. 10th Symposium on Combinatorial Pattern Matching (CPM99), volume 1645 of $L N C S$, pages 281-293. Springer-Verlag, 1999.

13. M. Zuker. RNA folding. Meth. Enzymology, 180:262-288, 1989. 